\title{
Synthesis Growth and Characterization of a Novel Non-Linear Optical Crystal: Cadmium Mercury Thiocyanate Dimethylsulphoxide
}

\author{
S.Cynthia ${ }^{1,}$ B.Milton Boaz ${ }^{2 *}$ \\ ${ }^{I}$ Department of Physics, Loyola College, Chennai, Tamilnadu, India. \\ $*^{2}$ Department of Physics, Presidency College, Tamilnadu, India.
}

\begin{abstract}
Single crystals of cadmium mercury tetrathiocyanate dimethylsulphoxide $\left[\mathrm{CdHg}(\mathrm{SCN})_{4}\left(\mathrm{H}_{6} \mathrm{C}_{2} \mathrm{OS}\right)_{2}\right], \mathrm{CMTD}$ was conveniently grown from a DMSO-water mixed solvent by slow evaporation technique. The good quality single crystal has been harvested in a period of 40-45 days. Different characterization studies have been carried out for finding its suitability in device fabrications. The lattice parameters of the crystal have been studied by single crystal X-ray diffraction analysis and the crystal system was identified as Orthorhombic. The Fourier transform infrared spectrum of CMTD has been recorded in the region 450-4000 $\mathrm{cm}^{-1}$ to identify the presence of functional groups. The optical studies have been carried out and it was found that the tendency of transmission observed from the specimen with respect to the wavelength of light is practically more suitable for opto-electronic applications. The photoconductivity studies confirm that the title compound has negative photoconducting nature. The relative second harmonic generation efficiency of the crystal has been tested by Kurtz-Perry powder technique.
\end{abstract}

Keywords: organometallic, non-linear optical crystals, characterization, synthesis, X-ray diffraction, dimethylsulphoxide

\section{Introduction}

The synthesis of organometallic materials with very good second order non-linearities have gained much attention in recent years. The need to develop these materials has gained prominence because of their inherent applications in the field of laser remote sensing, optical information processing, optical data storage etc. The present study focusess on non-linear optical crystals which finds its importance in opto-electronic applications. Cadmium mercury tetrathiocyanate dimethylsulphoxide (CMTD) is a useful candidate of SONLO(second order non-linear optical) crystal with an empirical formula $\mathrm{C}_{8} \mathrm{H}_{12} \mathrm{CdHgN}_{4} \mathrm{O}_{2} \mathrm{~S}_{6}$. The crystal structure is reported to be Orthorhombic with space groupP $2_{1} 2_{1} 2_{1}$. Here the growth of crystal was achieved by slow evaporation technique. We report the growth of CMTD single crystals of relatively large size $\left(17 \times 12 \times 3 \mathrm{~mm}^{3}\right)$. The grown crystals are characterized by XRD, NLO test, FTIR, Optical absorption studies, microhardness, dielectric and photoconductivity studies. The NLO property of the single crystal has been confirmed by SHG test. As part of continuous effort we report here the synthesis, structure and properties of a new complex crystal, CMTD.

\section{Experimental Details}

The commercially available potassium thiocyanate, cadmium chloride, mercury chloride (AR grade) have been used to synthesize the CMTC crystal. The growth and preparation have been carried out in aqueous solution to give the following reaction:

\section{$\left.4 \mathrm{KSCN}+\mathrm{Cdcl}_{2}+\mathrm{HgCl}_{2}---->\mathrm{CdHg}_{(\mathrm{SCN}}\right)_{4}+\mathrm{KCl}$}

The solution was filtered twice to remove dust particles and undissloved materials. By using dimethylsulphoxide as ligand and de-ionized water to react with Cadmium mercury thiocyanate(CMTC), seed crystals of CMTD were obtained. The chemical reaction is:

$$
\mathrm{CdHg}(\mathrm{SCN})_{4}+2\left(\mathrm{CH}_{3}\right)_{2} \mathrm{SO}--\cdot---\cdot>\mathrm{CdHg}(\mathrm{SCN})_{4}\left(\mathrm{H}_{6} \mathrm{C}_{2} \mathrm{OS}\right)_{2}
$$




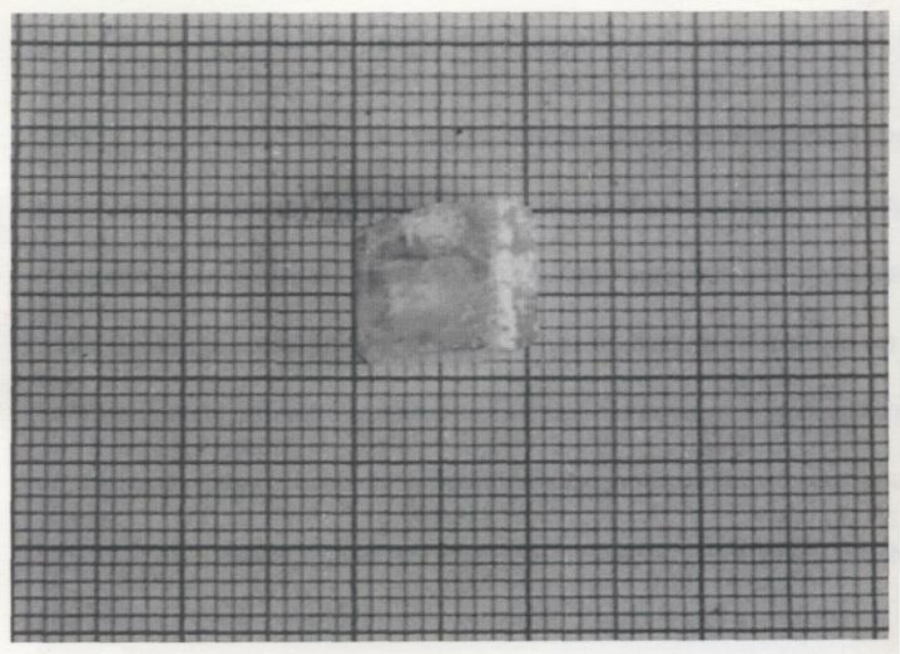

Fig1. Photograph of as grown CMTD single crystal

The single crystal X-ray diffraction analysis of crystals were carried out using Enraf Nonius CAD4 single crystal X-ray diffractometer. The structure was solved by direct method and refined by full matrix least square technique using SHELXL program. The lattice parameters and cell volume were determined. FTIR spectrum of the grown crystal was recorded in the range $4000-400 \mathrm{~cm}^{-1}$ using the instrument Bruker IFS-66V FTIR spectrometer. The optical absorption spectrum was recorded in the wavelength range 200-2000 nm using VARIAN CARY 5E spectrophotometer. The measurements of photocurrent and dark current were carried out using a picoammeter (Keithley 480). A well polished sample was attached to a microscopic slide on which two copper electrodes were fixed at a known distance using silver paint. The sample was connected to a dc power supply and a picoammeter. After shielding the sample from all radiation, the applied voltage was increased from 0 to $400 \mathrm{~V}$ in steps of $50 \mathrm{~V}$ and the corresponding dark currents were noted. In the second stage, the photocurrent of the same sample was measured by exposing it to radiation from a $100 \mathrm{~W}$ halogen lamp containing iodine vapour and tungsten filament. The experiment was repeated for different intensities of light. The dielectric constant of the sample was measured using HIOKI 353250 LCR HITESTER in the frequency range $50 \mathrm{~Hz}-$ $5 \mathrm{MHz}$. The second harmonic generation test was made using Kurtz and Perry technique.

\subsection{Single crystal XRD}

\section{Results and Discussion}

The lattice parameters and the crystal system have been determined using the single crystal X-ray diffraction analysis. The observed results indicated that the crystal belongs to orthorhombic crystal system with space group $\mathrm{P} 22_{1} 2_{1} 2_{1}$ and the determined unit cell parameters are $\mathrm{a}=8.0122 \AA, \mathrm{b}=8.6421 \AA$, $\mathrm{c}=28.854 \AA$, $\alpha=$ $90^{\circ}, \beta=90^{\circ}, v=90^{\circ}, V=2078 \AA^{3}$ respectively. The XRD data of the sample well coincides with the reported work ( Shiyi Guo et al 2000b).

\subsection{Second order non-linear optical property test}

The NLO efficiency of the crystal was estimated by using Kurtz and Perry technique and the efficiency of the sample was compared with microcrystalline powder of KDP and urea as the reference material. A Q-switched, mode locked Nd:YAG laser operating at $1.06 \mathrm{um}$ and generating pulses of $8 \mathrm{~ns}$ generating 6 $\mathrm{mJ} /$ pulse was allowed to pass through the crystal. The SHG was confirmed by emission of green radiation of the sample. The well known NLO crystals are taken as reference materials and the conversion efficiency of CMTD was compared. The results indicate that the efficiency of frequency doubling in CMTD crystal (75\%) is better than urea $(5 \%)$ and $\operatorname{KDP}(3 \%)$ crystals.

\subsection{FTIR Analysis}

The functional groups presented in the title compound have been identified by Bruker IFS 66V model FTIR spectrometer using KBr pellet technique in the region $450-4000 \mathrm{~cm}^{-1}$. The infrared absorption spectrum of CMTD crystal is shown in fig 2. In the high energy region, there is a broad band between 3500 and $2200 \mathrm{~cm}^{-1}$. The $\mathrm{CN}$ stretching vibration occurs above $2105 \mathrm{~cm}^{-1}$. On the other hand the CS stretching vibration lies between $870-790 \mathrm{~cm}^{-1}$ or $730-680 \mathrm{~cm}^{-1}$. The SCN bending vibration shows the $\mathrm{N}$ bonding near $470 \mathrm{~cm}^{-1}$ (Wang et al 2001;Nakamoto 1977). The CN stretching vibration implies that the strong band is at $2195 \mathrm{~cm}^{-1}$. The CS stretching vibration shows that the weak and sharp bands are at $710 \mathrm{~cm}^{-1}$ and $676.6 \mathrm{~cm}^{-1}$. The absorption peaks 
assigned to S-O stretching modes appear at lower frequency comparing with those of DMSO due to coordination of $\mathrm{O}$ atoms with the $\mathrm{Cd}$ atoms, which cause weaker bonds between $\mathrm{O}$ and $\mathrm{S}$ in the coordinated DMSO molecule. The stretching and bending modes of $\mathrm{CH} 3$ are found shifted from the frequencies of free DMSO. Therefore it is concluded that the molecular structure of DMSO in title compound is different from that of the free DMSO due to the fact that the DMSO molecule combines with $\mathrm{Cd}$ as a monodentate ligand through the $\mathrm{O}$ atom. Hence it may be stated that the introduction of DMSO ligand which contains the $\mathrm{S}=\mathrm{O}$ double bond improves the electron transferring ability and induces greater macroscopic non-linearity. The assignments of IR absorption of CMTD is presented table1 .

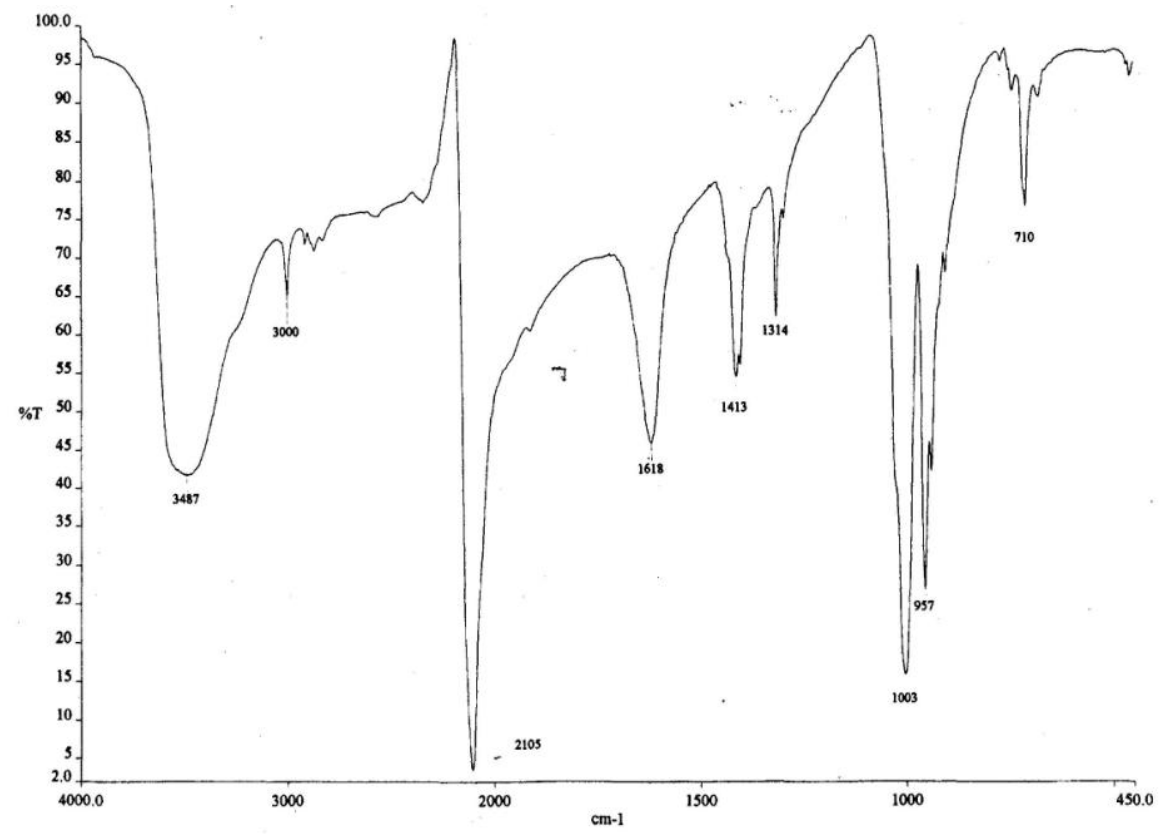

Fig2. FTIR spectrum of CMTD Crystal

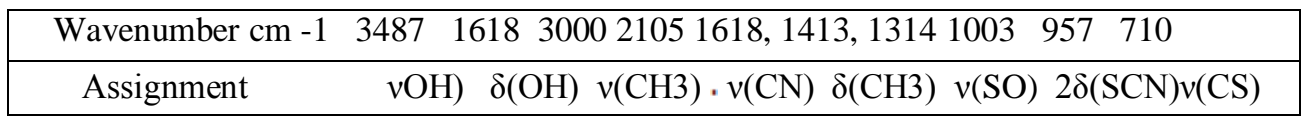

Table1. FTIR spectral data of CMTD

\subsection{Optical absorption spectrum}

Since single crystal is mainly used in opto-electronic applications, the optical transmission range and the transparency cut-off wavelength are essential. Optical behavior of CMTD was measured by Varian Cary 500 Spectrophotometer in the wavelength range200-2000 nm. The recorded spectrum is shown in fig.3. From this measurement, it is observed that the crystal is transparent in the wavelength range $200-1500 \mathrm{~nm}$ and the maximum absorbance is about $230 \mathrm{~nm}$. In the entire visible region the absorbance is very low, even less than 2 units.

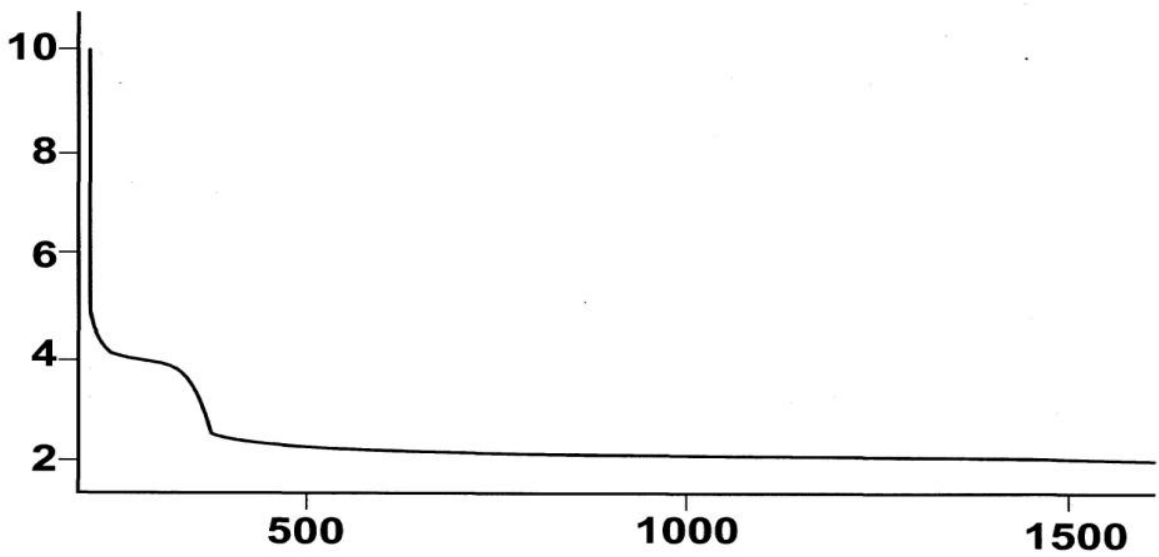

Fig3. Optical absorption spectrum of CMTD 


\subsection{Microhardness studies}

Microhardness studies were carried out on CMTD crystals for the applied load of 10 to $100 \mathrm{~g}$. The Vicker's hardness number (Hv) of CMTD is calculated using the relation

$\mathrm{Hv}=1.8544 \mathrm{p} / \mathrm{d} 2 \mathrm{~kg} \mathrm{~mm}-2$

Where $\mathrm{p}$ is the indentor load in $\mathrm{Kg}$ and $\mathrm{d}$ is the diagonal length of the impression in $\mathrm{mm}$. The values of $\mathrm{Hv}$ for various loads are plotted and shown in fig4. The graph indicates that the microhardness number decreases with increase in applied load. The decrease in microhardness number with the increasing load satisfies normal indentation size effect(ISE). By plotting applied load vs Vicker's hardness, the value of work hardening coefficient of CMTD was found to be 1.25 which indicates that the material belongs to the category of hard materials.

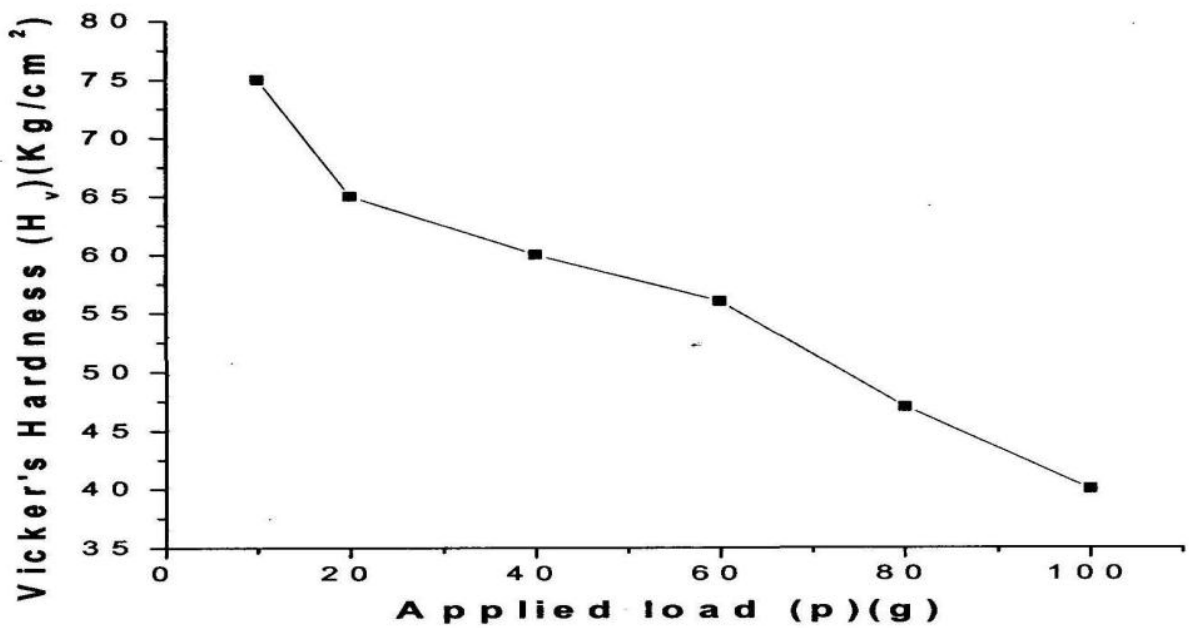

Fig4. Vickers hardness profile of CMTD as a function of applied load

\subsection{Dielectric studies:}

In the present work dielectric measurements are carried out using HIOKI 3532-50 LCR Hitester in the frequency range from $50 \mathrm{~Hz}$ to $5 \mathrm{MHz}$. The experiment is performed with defect free samples which are cut and later polished using fine grade alumina powder to obtain good surface finish. Good quality crystal was selected for dielectric studies. The dielectric constant was calculated using the relation

$$
\varepsilon^{\prime}=\frac{\mathrm{Cd}}{\mathrm{As}_{0}}
$$

where $\mathrm{C}$ is the capacitance, $\mathrm{d}$ is the thickness and $\mathrm{A}$ is the area of the sample. The plot of dielectric constant versus $\log$ of frequency is shown in Figure5.It is observed that the dielectric constant decreases with increase in frequency. Further at high frequency, the dipolar orientation effect is dominant whereas at low frequencies below $10 \mathrm{KHz}$ ionic and electronic polarization are effective. The large values of dielectric constant at low frequency enumerate that there is a contribution from all four known sources of polarization, namely electronic, ionic, dipolar and space- charge polarizations. Space charge polarization is generally active at lower frequencies and high temperatures. Further the space charge polarization is dependent on the purity and perfection of the material. The characteristics of low dielectric constant at high frequency suggest that the sample possesses enhanced optical quality with fewer defects. 


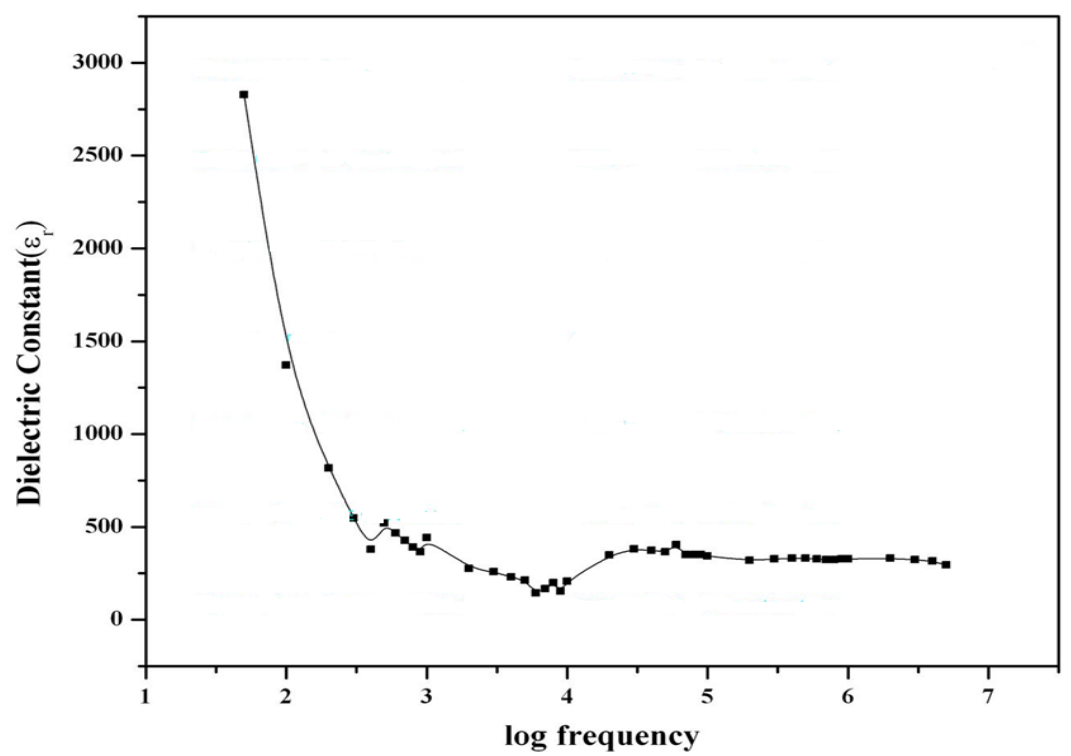

Fig5. dielectric constant vs log of frequency

\subsection{Photoconductivity studies}

Fig 6. Shows the variation of both dark current and photocurrent with applied field at different levels of illumination respectively. It is seen from the plots that both dark current and photocurrent of the sample increase linearly with the applied field and the photocurrent is seen to be less than the dark current for the same applied field. However, in all the cases, the strength of the photocurrent is found to be less than the dark current. The negative photoconductivity exhibited by the sample may be due to the reduction in the number of charge carriers in the presence of radiation (Bube 1981, Pandi and Jayaraman 2001). According to the Stockmann model, negative photoconductivity is based on two-level scheme. One level is located between the Fermi level and the conduction band, while the other is situated close to the valence band. Due to strong capture cross sections, recombination of electrons and holes takes place.. It may be attributed to the more positive charge of the metal ions.

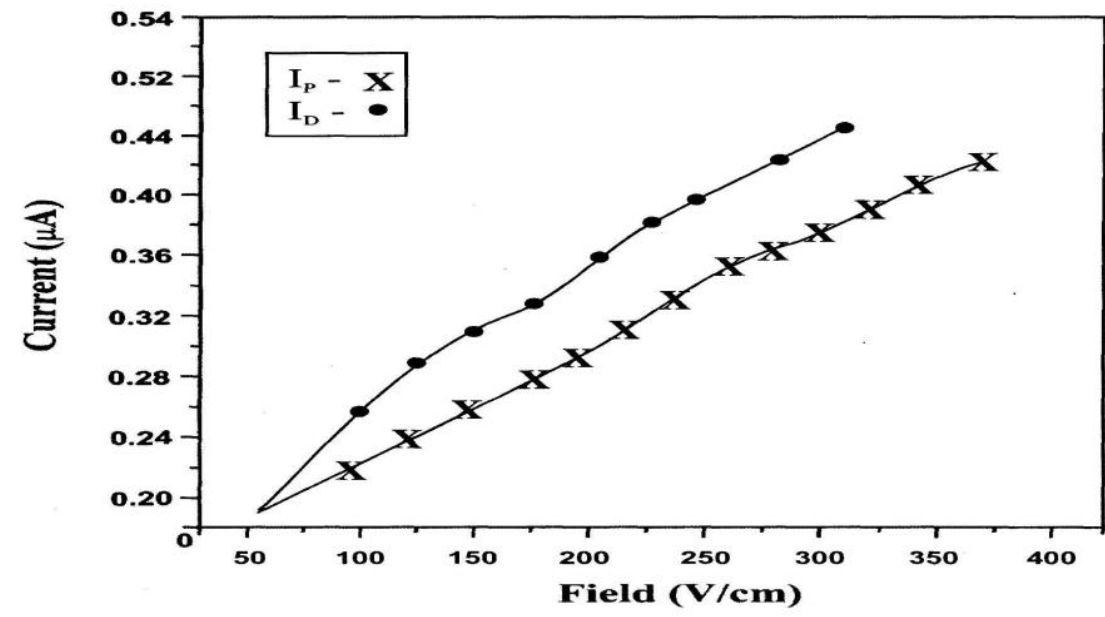

Fig6. Field dependent photoconductivity of CMTD single crystal 


\section{Conclusion}

The compound CMTD has been sysnthesized and the single crystal of CMTD were grown successfully in DMSO-water mixed solvent using the slow evaporation technique. The cell parameters were determined using single crystal X-ray diffraction method. Functional groups were obtained using FTIR analysis, which revealed the characteristic vibration modes of CMTD crystal. The optical property of the grown crystal was studied by Uv-Vis-NIR spectroscopy and the UV cut off wavelength of the grown crystal was about $230 \mathrm{~nm}$. The crystal possesses good optical transparency in the entire visible region. The microhardness study indicates that the CMTD crystals belong to the class of hard materials. The photoconductivity study confirms the negative photoconductivity nature of the sample. The dielectric studies indicate that CMTD has low value of dielectric permitivitty which explains its high SHG efficiency.

\section{Acknowledgement}

The authors are thankful to the Management of Loyola college and Presidency college for providing various facilities needed for this study. The authors express their sincere thanks to SAIF, IIT Chennai for the spectral facilities required for this purpose.

\section{References}

[1]. Wang X Q, Xu D, Lu M K, et al., J Crystal growth, 245(2002) 126.

[2]. Tian Y P, Duan C Y, Zhao C Y et al., Inorg chem., 36 (1997) 1247.

[3]. Sun H Q, Yuan D R, Wang X Q et al., J Crystal growth, 256 (2003) 183.

[4]. Duorong Yuan, Zhenwu Zhong, Mingguo Liu et al., J Crystal growth, 186 (1998) 240.

[5]. $\quad$ Kurtz S K \& Perry T T, J Appl Phys, 39 (1968) 3798.

[6]. Lewis J, Nyholm R S \& Smith P W, J Chem Soc, (1961) 4590.

[7]. Joshi V N, Photoconductivity, (Marcel Dekker, New York) 1990

[8]. Bube R H, Photoconductivity of solids, New York. 1981.

[9]. Pandi S \& D Jayaraman, Mater Chem Phys, 71(2001) 314.

[10]. Joseph G P and Rajarajan K, et al., J Crystal growth, 296 (2006) 1066.

[11]. Wang X Q, Xu D, Cheng X F \& Huang J, J Cryst growth, 271 (2004) 120

[12]. $\quad$ Bhat Govinda \& Dharmaprakash S M, Indian J Pure \& Applied Phys, 41 (2003) 627.

[13]. Ambujam K, Rajarajan K, Selvakumar S, et al., Indian J Pure \& Applied Phys, 44 (2006) 243.

[14]. Sebatini A \& Bertini I, Inorg Chem, 4 (1965) 959.

[15]. Raghavan C M, Sankar R, Mohankumar R \& Jayavel R, J Cryst Growth, 311 (2009) 1346. 